\title{
Beat around the bush for VA-LRTI
}

\author{
Ignacio Martin-Loeches ${ }^{1,2,3^{*}}$ (B), Pieter Depuydt ${ }^{4}$ and Michael S. Niederman ${ }^{5}$
}

๑ 2018 Springer-Verlag GmbH Germany, part of Springer Nature and ESICM

Nosocomial infection poses an important threat to patient outcome and puts a major burden on healthcare resources. The lower respiratory tract accounts for the majority of these infections, and patients admitted to the intensive care unit (ICU) are at the highest risk, especially when mechanical ventilation through an endotracheal tube is required. Ventilator-associated lower respiratory tract infection (VA-LRTI) consists of ventilator-associated pneumonia (VAP) and ventilator-associated tracheobronchitis (VAT) [1, 2]: a clinico-radiological distinction between the two is not always straightforward, and both conditions are probably on a spectrum of infection, with varying disease severity. Whereas VAP is associated with increased mortality (6-15\% of which is estimated to be attributable to VAP itself), this is less clear for VAT. Both VAP and VAT, however, are associated with increased healthcare costs and are major drivers of antibiotic prescription in the ICU, although the need to treat VAT with systemic antibiotics remains controversial (Fig. 1).

Microbial pathogens of VA-LRTI are frequently multidrug-resistant (MDR), which negatively affects patient outcome because of frequent inappropriate therapy, which further fuels antibiotic escalation and fosters more resistance. In an era of ever-increasing global antibiotic resistance and dwindling development of new antibiotic drugs, a healthcare crisis may be imminent, as expressed in a 2013 statement by Dame Sally Davies, England's Chief Medical Officer, saying that “...untreatable infection caused by antibiotic-resistant bacteria 'poses a catastrophic threat' to humans" and calls for immediate global action. [3].

In this respect, prevention of VA-LRTI is of the utmost importance. The grouping of a limited set of preventive

\footnotetext{
*Correspondence: drmartinloeches@gmail.com

${ }^{1}$ Multidisciplinary Intensive Care Research Organization (MICRO), St James's Hospital, Dublin, Ireland

Full author information is available at the end of the article
}

measures as a 'bundle' has been developed as a more structured approach to improve processes of care $[4,5]$. The goals, objectives and evaluation of bundles of care include developing implementation strategies, monitoring adherence to bundle elements and evaluating the impact of utilization. Key elements of VAP bundles are basic measures such as hand hygiene, head-of-bed elevation, avoiding routine change of ventilator circuits, minimizing sedation and making daily efforts at ventilator weaning.

In an article recently published in Intensive Care Medicine, Landelle and colleagues present the results of a study investigating the effects of a multifaceted prevention program on the incidence of VAP [6]. This prevention program consisted of an educational package focusing on nine interventions reflecting American Thoracic Society/Infectious Diseases Society of America (ATS/IDSA) guidelines, the introduction of continuous subglottic secretion aspiration at a later time point, and finally, the application of selective oral decontamination (SOD). The design was that of a before-after study, with a pre-intervention, intervention and post-intervention period, each lasting 8-11 months. The authors observed a significant reduction in VAP rates, and there was also a reduction in broad-spectrum antibiotic use for LRTI, although this broad category included community-acquired as well as nosocomial infections, and it is unclear which respiratory infections were included in this assessment. Remarkably, following the addition of SOD, VAP rates dropped to $70 \%$ of baseline, after an initial decrease to $42 \%$ after an eightelement bundle that already included oral chlorhexidine.

The strengths of the study are the measures of compliance with the bundle's components throughout the study and a thorough statistical analysis. However, several methodological limitations are present. Firstly, the study was performed in a single center, limiting its generalizability. Secondly, and more importantly, the study was essentially unblinded, and thus VAP diagnosis could have been subject to bias. Using quantitative cultures of invasively obtained alveolar samples [e.g., by bronchoalveolar

\section{Springer}


Compartmentalized response vs. binary approach in respiratory infections in critically ill patients.

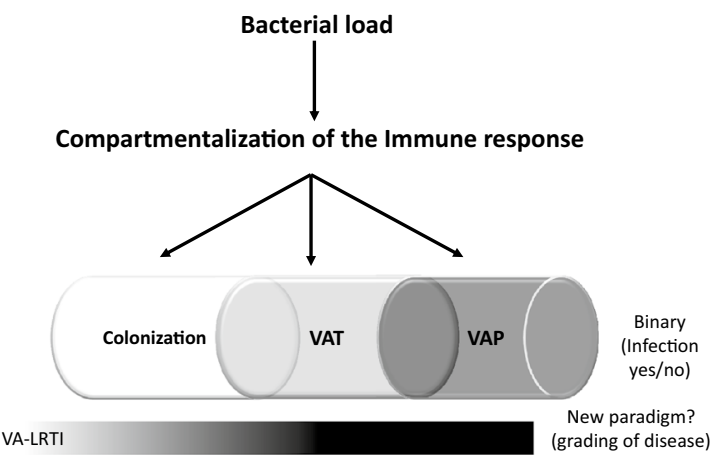

Fig. 1 .

lavage (BAL)] improves the precision of VAP diagnosis, essentially by increasing specificity, but this was not always done in the current study. Moreover, bias in sensitivity may have occurred, as proceeding to BAL is in practice always triggered by a subjective clinical suspicion of pneumonia. Finally, it cannot be ruled out that the sensitivity of definite VAP diagnosis was lower following the introduction of SOD, as SOD antibiotics may have been aspirated into the lung, interfering with culture recovery of bacteria.

In addition, it is somewhat puzzling that the low compliance with the bundle (only 60-67\% for hand hygiene, for example) would result in such a substantial reduction in VAP (incidence density rates of clinical VAP per 1000 ventilator-days at risk were $37.5,19.1$ and 15.7 in the preintervention, post-bundle, and post-SOD plus bundle periods, respectively), as this challenges the whole concept of 'all or nothing' that is inherent in bundle philosophy. Furthermore, despite the substantial reduction in the incidence of VAP and in the use of broad-spectrum antibiotics for LRTI, the intervention failed to achieve an overall reduction in antibiotic prescription in the ICU. The lack of a decrease in total antibiotic consumption during the study period could be due to a shift to or a dilution by other foci of nosocomial infection. Alternatively, there may have been an increased use of antibiotics for presumed respiratory infection that did not qualify for a diagnosis of LRTI (e.g., by lack of microbiological confirmation). One must be reminded again that labeling episodes as VAP or VAT is problematic, and if a drop in VAP was replaced by a rise in VAT, there may have been no meaningful reduction in LRTIs or in antibiotic use. In large epidemiological studies, $10-30 \%$ of patients with VAT progress to VAP, and VAT itself can lead to adverse outcome, meaning that if VAT is not eradicated, patient outcomes may not be improved $[1,2]$.
As the concept of care bundles explicitly groups interventions as an 'all-or-nothing' package, the impact of individual components cannot be measured. Thus, the ATS/IDSA bundle used in the present study included the application of chlorhexidine for oral care, an intervention that could be directly harmful [7, 8]. The benefit-harm ratio of chlorhexidine is unclear, and the current European guidelines for hospital-acquired pneumonia do not issue a recommendation regarding its use [1]. Very recently, a retrospective, hospital-wide, observational cohort study of over 80,000 adult hospitalized patients found oral chlorhexidine to be significantly associated with increased mortality [9]. However, whether selective oral decontamination with topical antibiotics should replace chlorhexidine as a safer or more effective bundle component of oral care is still far from clear. The added reduction in VAP incidence after the stepwise introduction of SOD in the present study suggests a benefit, but the study period was too short to appreciate all ecological consequences of its use.

In the end, prevention of VA-LRTI is essential to avoid patient harm, and established methods such as ventilator bundles again prove their usefulness in the current study. However, with the antibiotic crisis looming, we are called upon to look further than current patient outcome, and should try to curb our overall use of antibiotics, to preserve their potential for future patients. Successful prevention of infection should translate into real reduction in antibiotic prescriptions, not just in differently labeling them.

\section{Author details \\ ${ }^{1}$ Multidisciplinary Intensive Care Research Organization (MICRO), St James's Hospital, Dublin, Ireland. ${ }^{2}$ Department of Pneumology, Respira- tory Institute, Hospital Clinic, Institut d'Investigacions Biomèdiques August Pi iSunyer (IDIBAPS), University of Barcelona, Barcelona, Spain. ${ }^{3}$ Centro de Investigaciones biomedicas En Red-Enfermedades Respiratorias (CibeRes CB06/06/0028)-ISCIII, Barcelona, Spain. ${ }^{4}$ Department of Intensive Care, Ghent University Hospital, Ghent, Belgium. ${ }^{5}$ Division of Pulmonary and Critical Care Medicine, Weill Cornell Medicine, New York, NY, USA.}

\section{Compliance with ethical standards}

\section{Conflicts of interest}

The authors declare that they have no conflict of interest.

Received: 11 June 2018 Accepted: 12 June 2018

Published online: 21 October 2018

References

1. Torres A, Niederman MS, Chastre J et al (2017) International ERS/ESICM/ ESCMID/ALAT guidelines for the management of hospital-acquired pneumonia and ventilator-associated pneumonia: guidelines for the management of hospital-acquired pneumonia (HAP)/ventilatorassociated pneumonia (VAP) of the European Respiratory Society (ERS), European Society of Intensive Care Medicine (ESICM), European Society 
of Clinical Microbiology and Infectious Diseases (ESCMID) and Asociación Latinoamericana del Tórax (ALAT). Eur Respir J 50:1700582. https://doi. org/10.1183/13993003.00582-2017

2. Martin-Loeches I, Povoa P, Rodriguez A et al (2015) Incidence and prognosis of ventilator-associated tracheobronchitis (TAVeM): a multicenter, prospective, observational study. Lancet Respir Med 3:859-868

3. https://www.independent.co.uk/life-style/health-and-families/healt h-news/antibiotics-resistance-apocalypse-warning-chief-medical-offic er-professor-dame-sally-davies-drugs-a7996806.html accessed 10th June 2018

4. Alvarez-Lerma F, Palomar-Martinez M, Sanches-Garcia M et al (2018) Prevention of ventilator-associated pneumonia: the multimodal approach of the Spanish ICU 'Pneumonia Zero' program. Crit Care Med 46:181-188. https://doi.org/10.1097/CCM.0000000000002736

5. Bouadma L, Deslandes E, Lolom I et al (2010) Long-term impact of multifaceted prevention program on ventilator-associated pneumonia in a medical intensive care unit. Clin Infect Dis 51:1115-1122. https://doi. org/10.1086/656737
6. Landelle C, Nocquet Boyer V, Abbas M et al (2018) Impact of a multifaceted prevention program on ventilator-associated pneumonia including selective oropharyngeal decontamination. Intensive Care Med. https:// doi.org/10.1007/s00134-018-5227-4

7. Klompas M, Speck K, Howel MD et al (2014) Reappraisal of routine oral care with chlorhexidine gluconate for patients receiving mechanical ventilation: systematic review and meta-analysis. JAMA Intern Med 174:751-761. https://doi.org/10.1001/jamainternmed.2014.359

8. Price R, MacLennan G, Glen J, SuDDICU Collaboration (2014) Selective digestive or oropharyngeal decontamination and topical oropharyngeal chlorhexidine for prevention of death in general intensive care: systematic review and network meta-analysis. BMJ 348:g2197. https://doi. org/10.1136/bmj.g2197

9. Deschepper M, Waegeman W, Eeckloo K et al (2018) Effects of chlorhexidine gluconate oral care on hospital mortality: a hospital-wide, observational cohort study. Intensive Care Med. https://doi.org/10.1007/ s00134-018-5171-3 